\section{The byssus threads of Pinna nobilis: A histochemical and ultrastructural study}

\author{
Andrea Diana, ${ }^{1}$ Marcella Reguzzoni, ${ }^{2}$ \\ Terenzio Congiu, ${ }^{3}$ Antonio Rescigno, ${ }^{1}$ \\ Federica Sollai, ${ }^{1}$ Mario Raspanti ${ }^{2}$ \\ ${ }^{1}$ Department of Biomedical Sciences, \\ University of Cagliari \\ ${ }^{2}$ Department of Medicine and Surgery, \\ Insubria University \\ ${ }^{3}$ Department of Surgical Sciences, \\ University of Cagliari, Italy
}

\begin{abstract}
The byssus of Pinna nobilis, the largest bivalve mollusc in the Mediterranean Sea, was investigated by histochemistry, immunohistochemistry, Transmission Electron Microscopy (TEM), Scanning Electron Microscopy (SEM) and Atomic Force Microscopy (AFM). At low magnification, the byssus threads appeared distinctively elliptical in cross-section, with a typical size approaching $50 \times 25$ micron and a featureless glassy appearance. Histochemical and immunohistochemical techniques confirmed the presence of elastic domains but the absence of collagen, which is known to be the main component in other molluscs. Ultrastructural analysis by TEM revealed the presence of at least two components within the thread, and an inner arrangement of straight, tightly packed longitudinal streaks. SEM observations while confirming the inner packing of straight, parallel subfibrils, suggested in the fracture surfaces the presence of unidentified substance which cemented together the same subfibrils and which was removed by exposure to extreme $\mathrm{pH}$ values. AFM micrographs added further evidence for the tight packing of subfibrils and provided some evidence of orthogonal, barely visible connecting structures. Finally, $\mathrm{HCl}$ or $\mathrm{NaOH}$ treatment left the subfibrils clean and free from any other component.
\end{abstract}

\section{Introduction}

The byssus is a tensile structure present in the larval stage of all bivalve molluscs. ${ }^{1}$ In some cases it is retained as a neotenic character reaching up to few centimetres in length that consists of slender threads below millimetre in diameter. Notably, it is able to extend from the mollusc foot to an adhesive plaque, therefore making possible to anchor the same fibrils to the substrate. In particular, most of the available studies have focused on the adhesive plaque, whose ability to form a lifelong, extremely strong bond underwater would have a remarkable impact in specific fields such as chemistry, engineering and surgery. ${ }^{2} \mathrm{By}$ contrast, research data on the byssus threads are both relatively sparse and mostly dealing with one or two species of molluscs, where for obvious reasons the mytiloids have pride of place: above all Mytilus edulis, ${ }^{3-11}$ M. galloprovincialis, ${ }^{8,12}$ M. californianus, ${ }^{11,13}$ and $M$. viridis, ${ }^{14}$ but also more unusual species such as Geukensia demissa and Modiolus modiolus $^{9,11}$ or Bathymodiolus termophilus. ${ }^{9}$ Recent studies on non-mytiloids include Atrina rigida and Ctenoides mitis, ${ }^{15}$ the giant clam Tridacna maxima ${ }^{16}$ and the freshwater zebra mussel Dreissena polymorpha. ${ }^{2,6,17}$ In addition to mytiloids, an older study includes also Pinctada alba, Anomia ephippium, Congeria cochleata, Venerupis pullastra and Pinna nobilis ${ }^{18}$ with the last one as favoured species in several older papers, ${ }^{19-21}$ but apparently no scientific report has been published on the Pinna byssus since 1970 . The only recent works on this topic are a workshop contribution tightly focused on the cultural heritage of this material, ${ }^{22}$ and a web page of the Natural History Museum in Basel (http://www.muschelseide.ch/en/biologie/b yssus/faseranalyse.html).

Such a gap of interest is indeed difficult to be justified because Pinna nobilis is the largest clam of the Mediterranean Sea, with a length often exceeding 1 meter. The byssus produced by this mollusc has several distinct properties: it lacks both the specialized adhesion discs and the proximal stem typical of mytiloids, and it is particularly fine and smooth, growing up to $20 \mathrm{~cm}$. From ancient times, it has been occasionally spun and woven as a rare and precious textile, under the name of sea-silk. However, the inclusion of Pinna nobilis in the 92/43/CEE Directive and in the subsequent 2006/105/CE Directive as a protected species has banned it from harvesting and consequently exploiting as a textile source, even though few manufactured products of sea-silk still survive in museums and private collections.

The present research examines for the first time the ultrastructure of the byssus coming from Pinna nobilis with an integrated approach of Light Microscopy (LM), Transmission Electron Microscopy (TEM), Scanning Electron Microscopy (SEM) and Atomic Force Microscopy (AFM).
Correspondence: Andrea Diana, Dipartimento di Scienze Biomediche, Università degli Studi di Cagliari, Cittadella Universitaria, 09042 Monserrato (CA), Italy.

Tel. +39.070.6754054/56 - Fax +39.070.6754003. E-mail:diana@unica.it

Key words: Byssus; Pinna nobilis; fibrous proteins; microscopy.

Contributions: AD, study design, immunohistochemical experiments, data analysis, manuscript completion; MRe, FS, histochemical experiments, data analysis; TC, MRa, SEM experiments, data analysis; AR, study conception, samples acquisition; MRe, TEM experiments, data analysis; MRa, SEM and AFM experiments, data analysis, manuscript drafting. All the authors have red and approved the manuscript prior to submission.

Conflict of interest: The authors have no conflict of interest to declare.

Received for publication: 21 February 2017 Accepted for publication: 19 October 2017.

Acknowledgments: The authors sincerely thank Miss Chiara Vigo for the generous gift of the byssus fibres and the University of Cagliari for financial support.

This work is licensed under a Creative Commons Attribution-NonCommercial 4.0 International License (CC BY-NC 4.0).

(C) Copyright A. Diana et al., 2017

Licensee PAGEPress, Italy

European Journal of Histochemistry 2017; 61:2779 doi:10.4081/ejh.2017.2779

\section{Materials and Methods}

Byssus fibres were kindly donated by Miss Chiara Vigo, the only person in Italy legally entitled to harvest it in the Sardinian sea from living Pinna nobilis molluscs. The fibres, approximately $10 \mathrm{~cm}$ long, were simply washed in seawater and then air dried. Both ends of the fibres were discarded and only the central portion was analysed.

Some fibres were cut into $10 \mu \mathrm{m}$-thick sections with a Leica (Wetzlar, Germany) CM1950 cryostat and the sections were subsequently stained with HaematoxylinEosin, Van Gieson trichromic and PAS solutions. A few sections were collected on a multiwell plate and processed for collagen and elastin content according to the following immunohistochemical protocol. They were treated with $4 \%$ skimmed milk in PBS in order to saturate unspecific immunogenic sites, then incubated overnight at $4^{\circ} \mathrm{C}$ with 
rabbit polyclonal anti-type I collagen antibody (ab292, dilution1:50; Abcam, Cambridge, UK) and mouse monoclonal anti-elastin antibody (BA-4, dilution 1:500; Abcam). The sections were then covered for 45 min with secondary anti-rabbit goat antibodies conjugated to alkaline phosphatase, and immediately after the immunoreaction was evaluated with naphtol-AS-MX-phosphate and Fast Red TR. All specimens were observed with Nikon (Tokyo, Japan) Optiphot-2 microscope and imaged with Nikon D3300 camera as $2800 \times 1867$ pixel, 24 bpp JPEG images.

Specimens intended for TEM were incubated overnight at $4{ }^{\circ} \mathrm{C}$ in $25 \mathrm{mM}$ sodium acetate buffer, $\mathrm{pH} 5.8$, containing $0.05 \%$ Cupromeronic Blue (Seikagaku Corp., Tokyo, Japan) plus $2.5 \%$ glutaradehyde and $0.1 \mathrm{M} \mathrm{MgCl} 2$, dehydrated in graded ethanol and embedded in Epon 812. Ultra-thin sections, obtained on an RMC MTX ultramicrotome (Boeckeler Instruments Inc., Tucson, AZ, USA) fitted with a standard diamond knife, were counterstained with uranyl acetate and lead citrate and observed on a FEI Morgagni TEM (FEI, Eindhoven, The Netherlands) equipped with an Olympus (Hamburg, Germany) SIS MegaView II CCD camera and operated at $80 \mathrm{kV}$. Images were directly obtained in digital format as grayscale TIFF files with a resolution of $1280 \times 1024$ pixels.

Part of the specimens intended for SEM and AFM observation was treated overnight with $1 \mathrm{~N} \mathrm{HCl}$ at room temperature. Another set of samples was processed overnight with $1 \mathrm{~N} \mathrm{NaOH}$ while other samples were left untreated. All specimens were dehydrated with graded ethanol and hexamethyldisilazane, whereas selected specimens were carefully fractured to expose their inner structure. The obtained fragments, mounted on appropriate stubs with conductive biadhesive tape, were gold-coated in an Emitech K225 apparatus and observed on a Philips (Eindhoven, The Netherlands) XL30-FEG field-emission scanning electron microscope operating at $7-10 \mathrm{kV}$. Images were directly obtained in digital format as grayscale TIFF files with a resolution of $1424 \times 968$ pixels.

Similar specimens, equally mounted on stainless steel stubs with bi-adhesive tape, were observed with a Digital Instruments (now Bruker Corp., Billerica, MA, USA) MultiMode atomic force microscope fitted with a Nanoscope IIIa controller and phase extender. All observations were carried out in air in intermittent contact (TM-AFM) at a resolution of $512 \times 512$ samples and a speed of $\approx 2 \mathrm{~Hz}$, using Nanoscope TESP-SS or Olympus OTESPA probes $\left(\mathrm{k} \approx 42 \mathrm{~N} \mathrm{~m}^{-1}\right.$

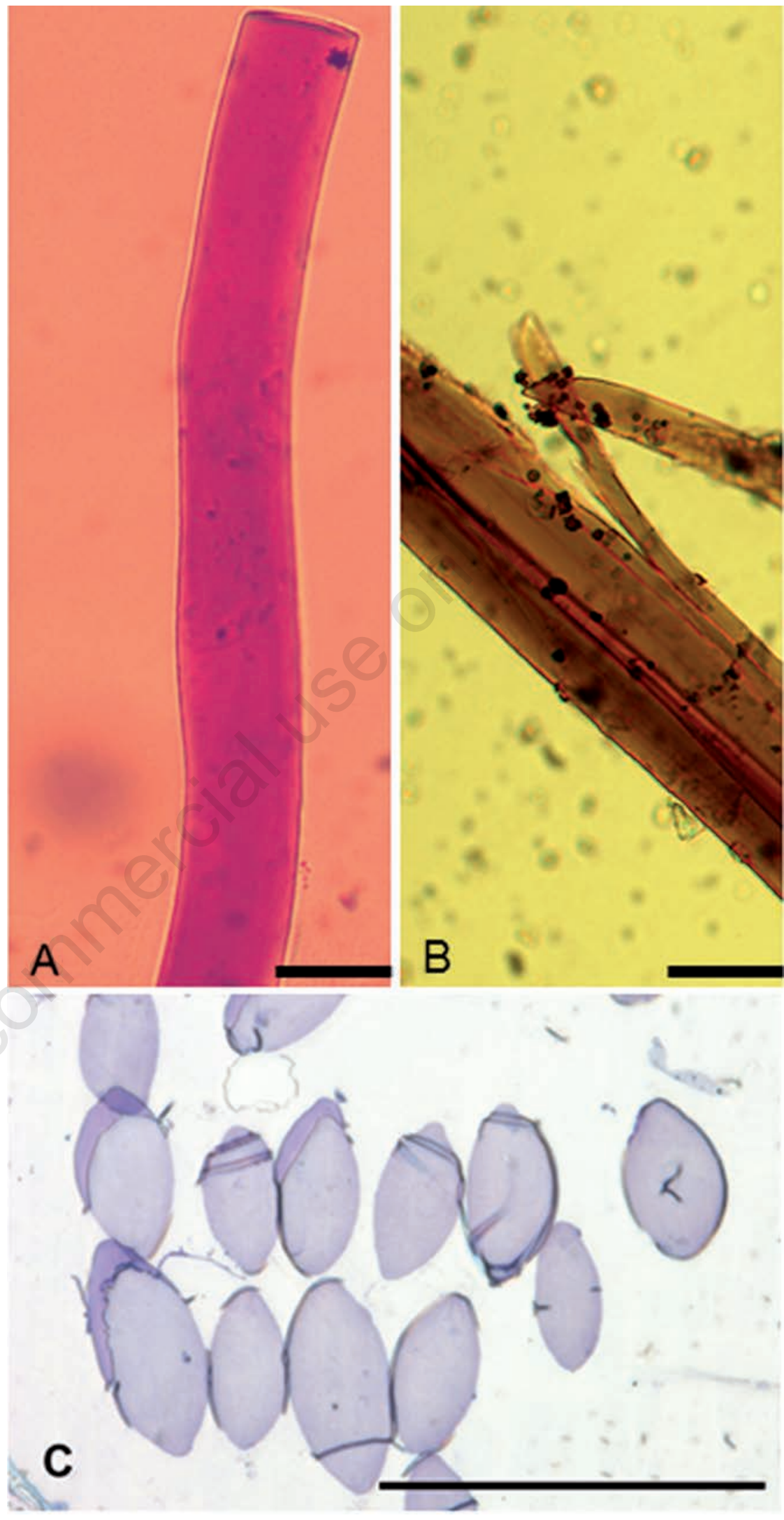

Figure 1. Micrographs of cryosectioned byssus fibres (A, B) and of resin-embedded semithin sections (C). The fibres are positive to PAS (A) and are stained dark brown by the Van Gieson trichrome staining (B). Semi-thin, toluidine-stained cross-sections show the distinctive elliptical shape of the fibres: see also http://www.muschelseide.ch/en/biologie/byssus/faseranalyse.html. Scale bars: A, B) $25 \mu \mathrm{m}$; C) $100 \mu \mathrm{m}$. 
and $\mathrm{f} \approx 300 \mathrm{kHz}$, for both).

When not otherwise specified, all chemicals and reagents were supplied by Sigma-Aldrich (St. Louis, MO, USA).

\section{Results}

Semithin sections of byssus fibres observed at the light microscope consistently showed a featureless, glassy appearance. Haematoxylin-Eosin staining had no visible effect (not shown), while the same fibres reacted positively both to PAS (Figure 1A), taking a distinct purplish red colour, and to the Van Gieson trichromic solution, which stained the fibres in dark brown (Figure 1B). Semithin cross-sections (Figure 1C) revealed a distinctive elliptical shape, with a greater axis of $30 \div 50 \mu \mathrm{m}$ and a minor axis of $20 \div 25 \mu \mathrm{m}$. Immunohistochemical reactions also gave different results, with a light but unmistakable reaction to anti-elastin antibodies and no evident immunodetection of collagen (Figure 2). In all experiments the results were completely consistent among different fibres.

The fibres intended for TEM analysis were embedded and sectioned along a known orientation. By means of TEM they still remained featureless at lower magnifications, and only from 14,000x they began to display a grainy, faint microfibrillar substructure oriented along the fibre (Figure 3A). Transected fibres revealed a mottled appearance made of two evident types of electron-dense sub-fibrils randomly intermixed (Figure 3B).

On the other hand, SEM micrographs were more informative about the outer features. In addition to a smooth and featureless surface, the untreated fibres exhibited occasional signs of extrusion in form of longitudinal, parallel streaks and ridges (Figure $4 \mathrm{~A})$, even though no distinct coating was detectable in cross-sections even at the highest magnifications. The inside of the fibres revealed a tight packing of longitudinal, parallel filaments (Figure 4B), but the fracture plane seemed to run unpredictably rather than along the filaments. Upon overnight treatment with $\mathrm{HCl}$ the general texture of the fibres confirmed the same arrangement of tightly packed, longitudinal thin filaments (Figure 5A) with a thickness of approximately $25 \mathrm{~nm}$ coupled to a grainy appearance. Moreover, fibre exposure to $\mathrm{NaOH}$ affected more dramatically the inner structure of the fibres, whose filaments became somewhat unbound, easily splitting longitudinally under a moderate flexion (Figure 5B) and flaring at the cut ends so that single filaments are clearly exposed (Figure 5C).

Atomic force micrographs were entirely consistent with the TEM and SEM pictures confirming that the fibres are made of a tight packing of longitudinal filaments (Figure 6A). The fracture surface ran both
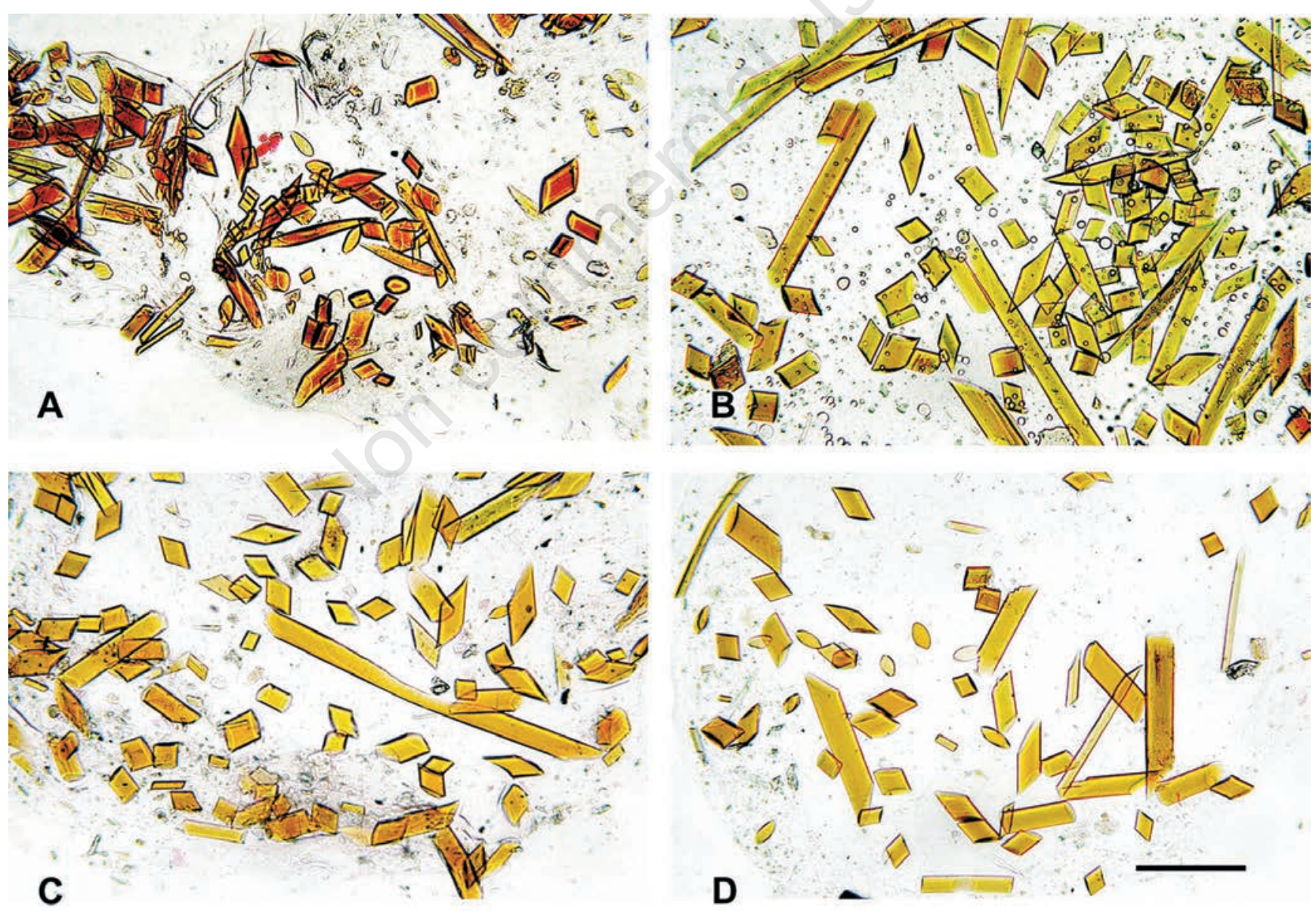

Figure 2. Micrographs of cryosectioned fibres after immunohistochemical treatment. Fibres are immunoreactive for elastin antibodies (A) compared to negative control (B). Fibres treated with anti-collagen antibodies (C) are not different from their negative control (D). Scale bar: $100 \mu \mathrm{m}$. 
along and across the filaments that occasionally appeared interconnected by slender transversal bridges (Figure 6B). Notably such orthogonal filaments were detectable only by AFM since in the SEM protocol all specimens need to be coated by a thin gold layer $(10-12 \mathrm{~nm})$ that is likely to mask the thinner structures. In addition, the filaments became cleaner and more evident after strong acid or alkali treatment (Figure 6C).

\section{Discussion}

From pioneering works on common mytiloids byssus, experimental approaches have been mainly focused on the biochemical characterization and elucidation of mechanical properties. So far, byssus from Mytilus edulis and galloprovincialis have been the most studied with regard to the presence of collagen that accounts for 3 different proteins covered by matrix proteins and additional sticky proteins. ${ }^{23}$ Collagen is indeed the most represented protein within the byssal threads with over $50 \%$ of the total content, ${ }^{24}$ although their collagen sequence is species specific. ${ }^{4}$ As a matter of fact, the homology with other mammalian collagens is limited to repeating triplets Gly-X-Y, wherein $\mathrm{X}$ is generally proline and $\mathrm{Y}$ is often hydroxyproline or hydroxylysine. The structural assembly of byssal threads in mytiloids is complicated by inconsistent presence of the hydroxylated forms that make difficult to figure out the correct three-dimensional folding of byssal elements. ${ }^{8,25}$ At variance, morphological and histochemical studies dealing with common or distinct features in byssus produced by several bivalve species have been limited to sparse data on ultrastructural composition and often referred to commercially interesting mytiloids but sufficient to rule out the banding patterns fibrillar collagen. ${ }^{26}$ As far as we know, this is the first systematic study that tries to combine results from ultrastructural investigations (TEM, SEM and AFM) and histochemical analysis of the byssus secreted by the biggest bivalve non mytiloid mollusc Pinna nobilis. In the present work, optical microscopy was limited to a rather basic approach since an old but excellent paper examined mainly the mucopolysac-
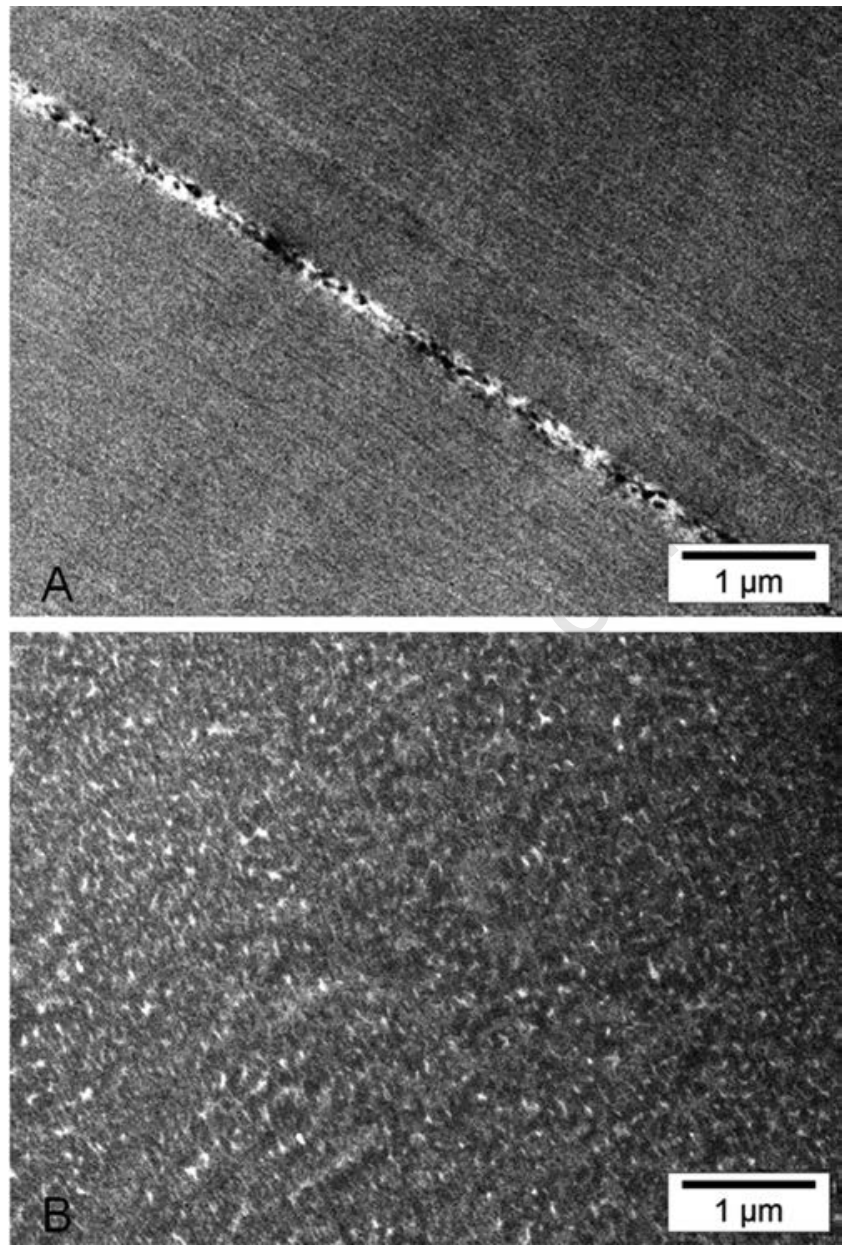

Figure 3. A) TEM micrograph of two adjoining fibres sectioned longitudinally; an ill-defined inner substructure, consisting of parallel microfibrillar material, is visible. B) TEM micrograph of a transected byssus fibre; the fibre appears to be a random mixing of two components of different electron-density. 14,000x magnification.
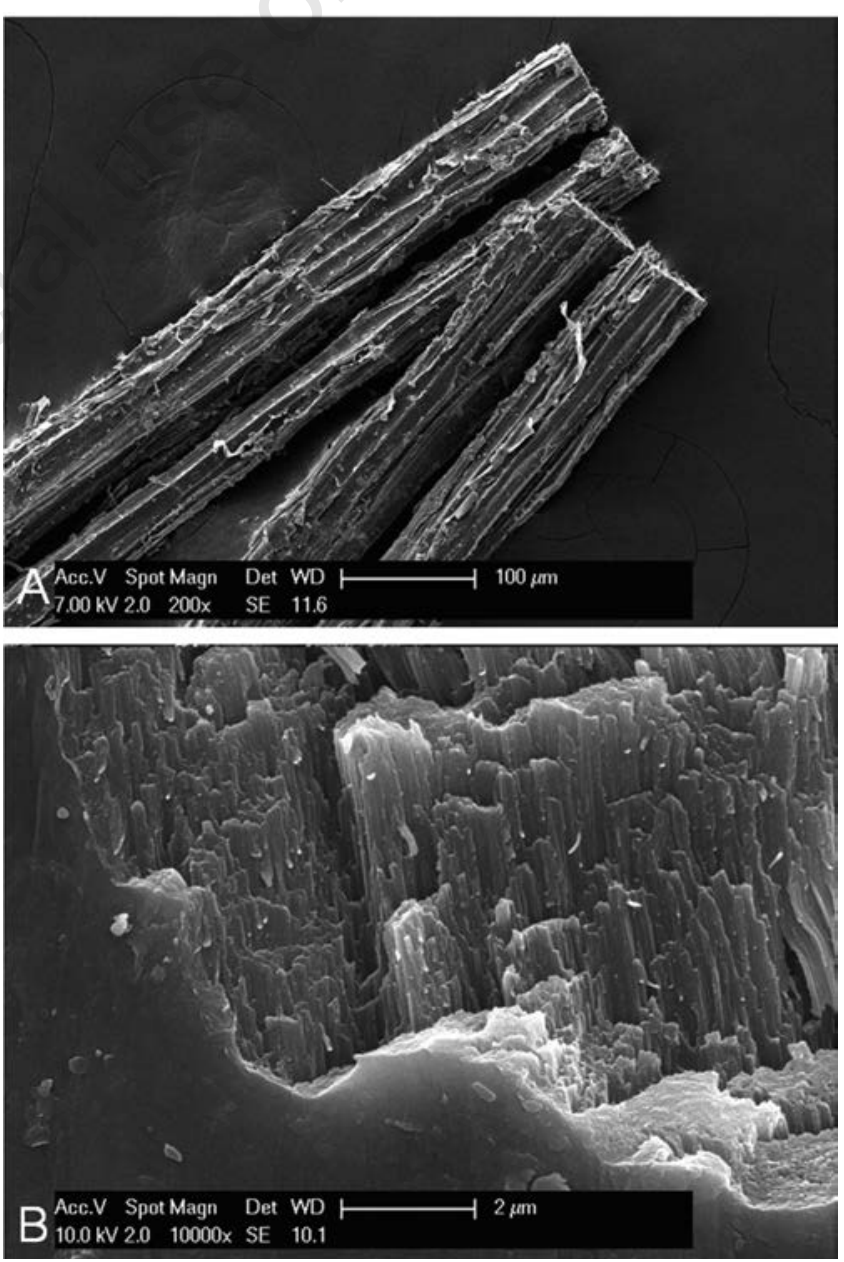

Figure 4. A) SEM micrograph of a bundle of fibres, covered by an amorphous coating marked by evident longitudinal extrusion streaks. B) High magnification detail of the fractured surface of a byssus fibre; in contrast with the smooth, featureless fibre surface (lower-left part of the picture), the fracture plane shows a tight packing of straight, parallel subfibrils; the fracture does not appear to follow a preferential plane. 
charidic and glycoproteic content of byssus related glands ${ }^{27}$ in Mytilus edulis and Pinna nobilis with some additional information about the histochemical nature of the same byssus threads. Therefore, the three histochemical stainings used in our study namely PAS, Van Gieson trichrome and toluidine were essentially confirmatory of the biochemical relationship between those gland secretions and byssus structure. Moreover, in order to ascertain the possible non colla- genic nature of the Pinna byssus we aimed to elucidate this matter by integrating morphological submicroscopic analysis and immunochemical detection of collagen I and elastin since, in contrast to the byssus of mytiloids, whose main components are "modified" collagens ${ }^{7,13}$ with fibrillar organization, molecular structure of Pinna byssus seems to be devoid of any classical collagen molecule. Indeed, its absence has been previously suggested by X-ray diffrac- tion $^{19-21}$ while a recent FT-IR analysis (http://www.muschelseide.ch/en/biologie/b yssus/faseranalyse.html) was in favour of a spectrum common to all animal fibrous proteins. Another recent paper only dealt with the fibres pigments. ${ }^{22}$

In addition, within other bivalve organisms such as Tridacna maxima, no evidence of collagen has been also documented within the produced byssus. The aforementioned findings, possibly related to a developmen-
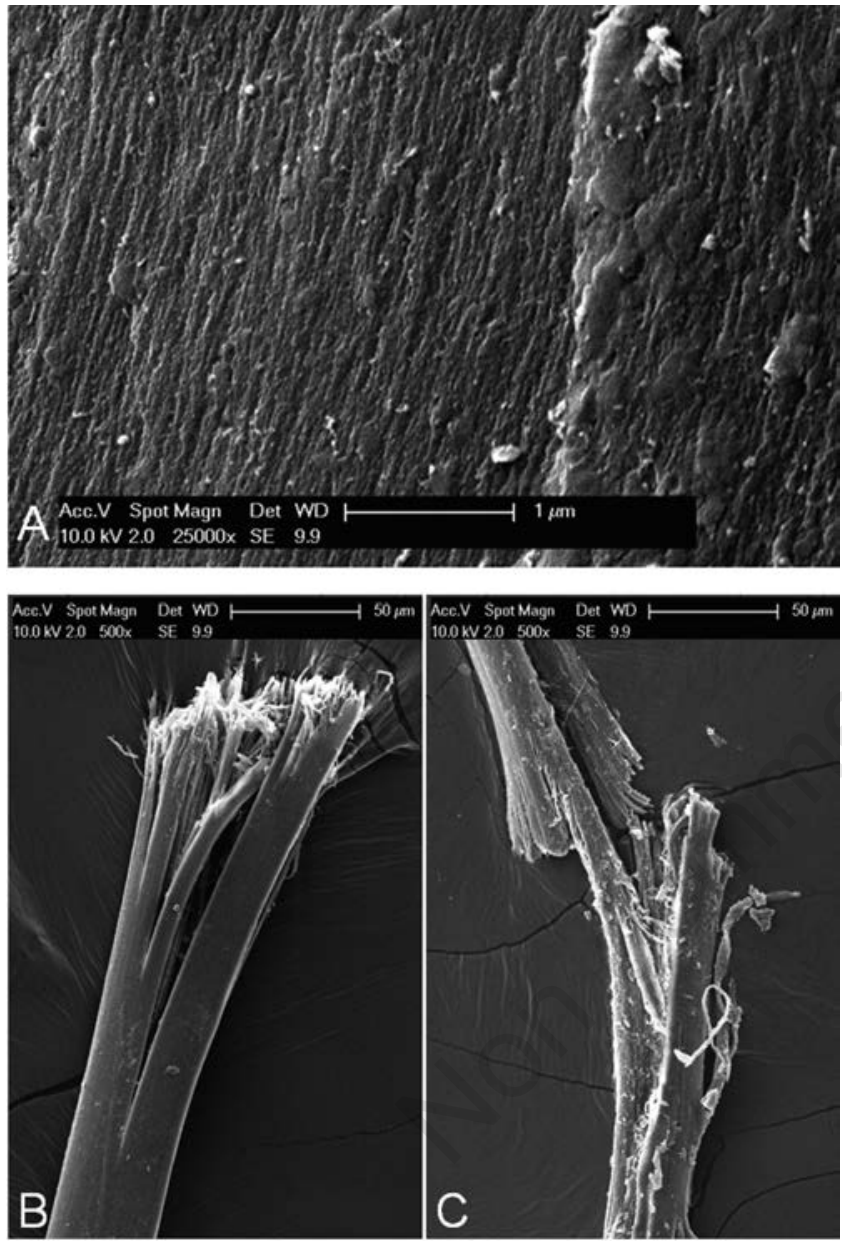

Figure 5. A) The surface of a fibre after overnight exposure to $1 \mathrm{~N}$ $\mathrm{HCl}$. The treatment has unmasked the underlying packing of straight, parallel filaments. B) Details of the flared, broom-like end of a $\mathrm{NaOH}$-treated fibril showing a number of longitudinal gaps; compare with the clear-cut ends of the untreated fibres of Figure 4A. C) The treatment with $\mathrm{NaOH}$ has weakened the mutual adhesion of the subfibrils to the point that these two fibrils broke and split after a moderate flexion.

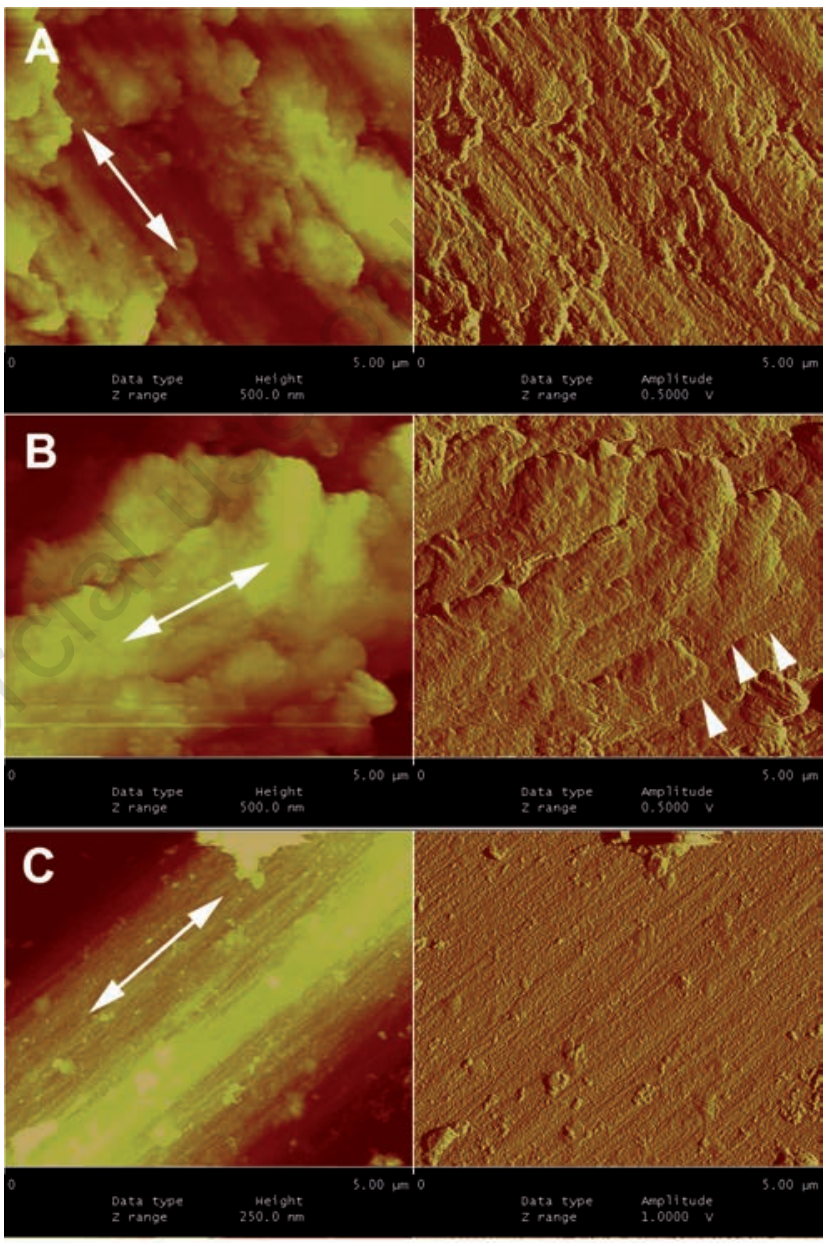

Figure 6. Dual-channel AFM pictures of the fracture surface of byssus fibrils. In each figure the left panel shows the Height channel and depicts the actual $3 \mathrm{D}$ topography of the specimen, where lighter colours corresponding to higher zones; the right panel shows the corresponding Amplitude channel, a feedback signal similar to the derivative of the Height and providing an alternative view of the same area. All micrographs have been intentionally taken so that the subfibrils run obliquely, to rule out possible artefacts that sometimes appear along the X-Y axes. A) Dualchannel AFM picture of a fractured untreated fibril; the arrows indicate the orientation of the subfibrils, better visible in the right panel. B) Another AFM micrograph of a fractured untreated fibre; thin, barely visible filaments (arrowheads) run orthogonal to the subfibrils. C) Same magnification of a byssus fibre after $\mathrm{HCl}$ treatment; the subfibrils, whose course is again marked by the arrows, are now cleaner and much more visible. In all panels the field of view spans $5 \times 5 \mu \mathrm{m}$. 
tal defect of the collagen-secreting gland or "white gland", ${ }^{24}$ definitely correlates with our immunohistochemical results that made possible to verify the presence of elastic domains by means of specific anti-elastin antibodies, ${ }^{6}$ but unexpectedly collagen-like negative pattern in the elastic flanking regions.

The first comparative biochemical analysis of Pinna byssus thread by means of amino acid analysis ${ }^{18}$ revealed hydroxyproline lack and glycine percentage about one third compared to Mytilus thread. ${ }^{16}$ Finally, Bouhlel et al. ${ }^{28}$ have unambiguously solved the multifaceted scenario from the overlapping of heterogeneous methodologies along the last decades by a rigorous comparison of mechanical and molecular properties of single species producing byssal threads. In that study, with regard to Pinna nobilis, the most striking finding from amino acid analysis, comes from the low content in glycine (around 14\%), and less than 5\% of alanine (glycine plus alanine less than 20\%). By contrast, the amino acid composition that accounts for $14 \%$ of proline, more than double compared to the other examined mytiloids, is quite intriguing whereas introduces a new question in terms of function. By the way, considering that the overall content of the mentioned amino acids does not reach $33 \%$, it becomes apparent that our negative results in the immune experiments for collagen I are in agreement with the special nature of collagen-like molecule possibly integrated into the byssus thread. Thus, we can reasonably draw the conclusion that byssus from several molluscs is variable in composition and in function across different species. On the basis of the experimental evidence from different microscopy techniques, it is well established that each byssus thread is a very compact fascicle of parallel, rectilinear filaments, containing at least two different components with different reactivity to heavy metal staining. In addition, our analysis carried out by SEM and AFM approach consistently confirmed that byssus threads have a thickness close to $50 \mu \mathrm{m}$ as already stated by Bouhlel report, ${ }^{28}$ but also that the same threads are composed of thin, straight and parallel filaments cemented by some other component - possibly a glycoconjugate as suggested by the PAS positivity - which is removed by $\mathrm{NaOH}$ and, to a lesser extent, by $\mathrm{HCl}$. It must be noted that, within these acidic or alkaline experimental conditions and in spite of their highly anisotropic architecture of longitudinal filaments, the fibres tend to snap along a transversal plane (therefore creating the least possible fracture area) rather than splitting along the filaments.
This suggests that in intact fibres strong inter-filament bonds are active, so that overlapping fibres functionally behave as an isotropic-like tissue. Only once these chemical bonds have been removed by strong alkali treatment, the filaments dictate the path of least resistance. It can be speculated that these inter-filament bonds correspond to the orthogonal filaments revealed only by AFM.

On the other hand, in the field of cultural heritage its susceptibility to extreme $\mathrm{pH}$ conditions, and especially strong alkali, should be of some importance for the preservation of the few and rare remaining manufactured products of sea-silk, witnesses of an age and a material certainly irreplaceable.

\section{References}

1. Yonge M. On the significance of the byssus in the bivalvia and its effects in evolution. J Mar Biol Ass UK 1962;42: 113-25.

2. Farsad N, Sone ED. Zebra mussel adhesion: structure of the byssal adhesive apparatus in the freshwater mussel, Dreissena polymorpha. J Struct Biol 2012;177:613-20.

3. Price HA. Byssus thread strength in the mussel, Mytilus edulis. J Zool Lond 1981;194:245-55.

4. Qin X, Waite JH. Exotic collagen gradients in the byssus of the mussel Mytilus edulis. J Exp Biol 1995;198:633-44.

5. Qin XX, Coyne KJ, Waite JH. Tough tendons. Mussel byssus has collagen with silk-like domains. J Biol Chem 1997;272:32623-37.

6. Coyne KJ, Qin XX, Waite JH. Extensible collagen in mussel byssus: a natural block copolymer. Science 1997; 277:1830-2.

7. Waite JH, Qin XX, Coyne KJ. The peculiar collagens of mussel byssus. Matrix Biol 1998;17:93-106.

8. Lucas JM, Vaccaro E, Waite JH. A molecular, morphometric and mechanical comparison of the structural elements of byssus from Mytilus edulis and Mytilus galloprovincialis. J Exp Biol 2002;205:1807-17.

9. Brazee SL, Carrington E. Interspecific comparison of the mechanical properties of mussel byssus. Biol Bull 2006; 211:263-74.

10. Aldred N, Wills T, Williams DN, Clare AS. Tensile and dynamic mechanical analysis of the distal portion of mussel (Mytilus edulis) byssal threads. J R Soc
Interface 2007;4:1159-67

11. Pearce T, LaBarbera M. A comparative study of the mechanical properties of Mytilid byssal thread. J Exp Biol 2009a;212:1442-8.

12. Hagenau A, Scheidt HA, Serpell L, Huster D, Scheibel T. Structural analysis of proteinaceous components in byssal threads of the mussel Mytilus galloprovincialis. Macromol Biosci 2009;9:162-8.

13. Harrington MJ, Gupta HS, Fratzl P, Waite JH. Collagen insulated from tensile damage by domains that unfold reversibly: In situ X-ray investigation of mechanical yield and damage repair in the mussel byssus. J Struct Biol 2009; 167:47-54.

14. Patel MB, Pithawala KA. Ultrastructural study of Mytilus viridis byssus through scanning electron microscopy. Int J Curr Res Aca Rev 2015;3:20-7.

15. Pearce T, LaBarbera M. Biomechanics of byssal thread outside the Mytilidae: Atrina rigida and Ctenoides mitis. J Exp Biol 2009;212:1449-54.

16. Miserez A, Li Y, Cagnon J, Weaver JC, Waite H. Four-stranded coiled-coil elastic protein in the byssus of the Giant Clam, Tridacna maxima. Biomacromolecules 2012;13:332-41.

17. Gilbert TW, Sone ED. The byssus of the zebra mussel (Dreissena polymorpha): spatial variations in protein composition. Biofouling 2010;26:829-36.

18. Pujol JP, Rolland M, Lasry S, Vinet S. Comparative study of the amino acid composition of the byssus in some common bivalve molluscs. Comp Biochem Physiol 1970;34:193-201.

19. Mercer EH. Observations on the molecular structures of byssus fibers. Aust J Mar Fresh Res 1952;3:199-204.

20. Jackson SF, Kelly FC, North ACT, Randall JT, Seeds WE, Watson M, et al. The byssus threads of Mytilus edulis and Pinna nobilis. In: JT Randall, SF Jackson, editors. Nature and structure of collagen. London: Butterworths; 1953. p. 106-16.

21. Rudall KM. The distribution of collagen and chitin. Symp Soc Exp Biol 1955;9:49-72.

22. Jaworski JS. Properties of byssal threads, the chemical nature of their colors and the Veil of Manoppello. Proc. Int. Workshop on the Scientific approach to the Acheiropoietos Images. ENEA Research Center Frascati, Italy.

23. Suhre MH, Gertz M, Steegborn C, Scheibel T. Structural and functional features of a collagen-binding matrix 
protein from the mussel byssus. Nat Commun 2014;5:3392.

24. Harrington MJ, Waite JH. Short-order tendons: liquid crystal mesophases, metal-complexation and protein gradients in the externalized collagens of mussel byssal threads. In: T Scheibel, editor. Fibrous proteins, Austin: Landes Bioscience; 2008. p. 30-45.

25. Rodriguez R, Moran L, Gonzales G, Troncoso E, Zuñiga RN. Collagen extraction from mussel byssus: a new marine collagen source with physicochemical properties of industrial interest. J Food Sci Technol 2017;54:122838.

26. Vitellaro-Zuccarello L, De Biasi S, Bairati A. The ultrastructure of the byssal apparatus of a mussel. V. Localization of collagenic and elastic components in the threads. Tissue Cell 1983;15:547-54.
27. Pujol JP. Le complexe byssogène des Mollusques bivalves. Histochimie comparée des sécrétions chez Mytilus edulis L. et Pinna nobilis L. Bull Soc Linn Normand 1967;8:308-32.

28. Bouhlel Z, Genard B, Ibrahim N, Carrington E, Babarro JMF, Lok A, et al. Interspecies comparison of the mechanical properties and biochemical composition of byssal threads. J Exp Biol 2017;220:984-94. 